\title{
DOENÇA DE CASTLEMAN SIMULANDO NEOPLASIA PANCREÁTICA
}

\section{CASTLEMAN DISEASE PRESENTINGAS PANCREATIC TUMOR}

\section{Antonio Cavalcanti de Albuquerque, TCBC-PE ${ }^{1}$ \\ Orlando Enedino da Silva Junior, ${ }^{2}$ Álvaro Antônio Bandeira Ferraz, TCBC-PE ${ }^{3}$}

\section{INTRODUÇÃO}

A doença de Castleman (DC), também conhecida como hiperplasia linfonodal gigante ou hiperplasia angiofolicular linfonodal foi descrita pela primeira vez em um relato de caso do Massachusetts General Hospital em 1954 pelo Dr. Benjamin Castleman ${ }^{1}$.

Usualmente a DC ocorre em adultos jovens e se apresenta como uma massa mediastinal assintomática ou adenopatia localizada, podendo abranger o tórax (63\%), pescoço (14\%), abdome $(11 \%)$ e axila $(4 \%)^{2}$. Caracteriza-se por ser um raro distúrbio linfoproliferativo, com evolução quase sempre benigna e etiologia desconhecida.

Neste relato descrevemos um caso de tumor de Castleman simulando neoplasia pancreática operado em nosso serviço.

\section{RELATO DO CASO}

Paciente do sexo masculino, branco, 33 anos, natural e procedente do Recife. Admitido com queixa de dor em flanco direito irradiada para o testículo há quatro meses. Referia ainda perda de quatro quilos neste período. $\mathrm{O}$ interrogatório sintomatológico e exame físico eram normais. Negava qualquer antecedente cirúrgico. Exames laboratoriais, incluindo marcadores tumorais (alfa-fetoproteina, antígeno carcinoembriogênico e antígeno carboidrato 19.9) encontravam-se dentro da normalidade. Radiografia do tórax sem alterações. A ultrassonografia de abdome
(USG) evidenciava massa hipoecóica de aproximadamente 6,0 x 3,0 cm na topografia da cabeça do pâncreas. A tomografia computadorizada do abdome (TC) revelou um processo expansivo sólido, homogêneo e em contiguidade com a cabeça do pâncreas sem dilatação do Wirsung ou da via biliar (Figura 1) A TC ainda mostrava um hemangioma hepático no segmento VI. A angiografia identificou uma massa hipervascularizada irrigada pelo tronco celíaco (Figura 2).

À laparotomia evidenciou-se a presença de massa de aproximadamente seis $\mathrm{cm}$ em seu maior diâmetro, de consistência elástica, bem circunscrita e contida na cápsula pancreática. O estudo histopatológico demonstrou hiperplasia angiofolicular linfonodal do tipo hialino-vascular.

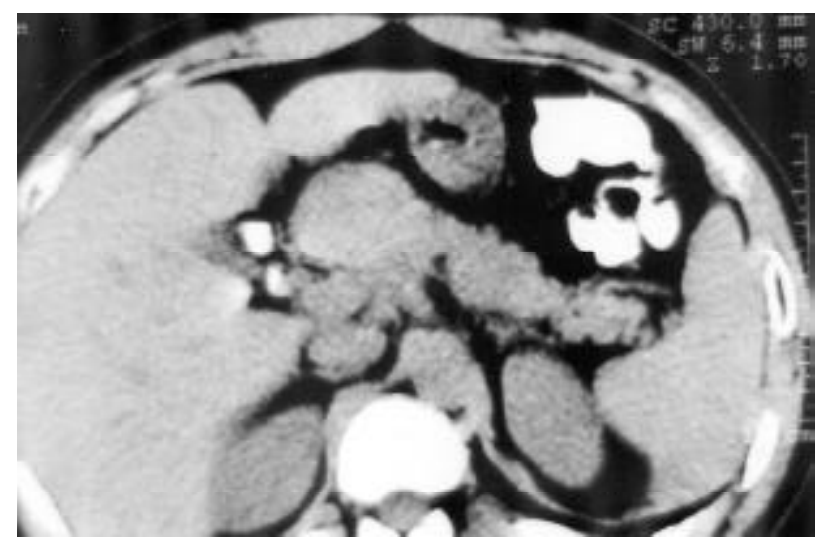

Figura 1 - TC do abdome demonstrando massa homogênea localizada na cabeça do pâncreas.

1. Mestrando em Cirurgia; Serviço de Cirurgia Geral da Universidade Federal de Pernambuco

2. Residente de Cirurgia Geral; Serviço de Cirurgia Geral da Universidade Federal de Pernambuco

3. Professor Adjunto do Departamento de Cirurgia da Universidade Federal de Pernambuco

Recebido em 22/10/2002

Aceito para publicação em 18/02/2003

Trabalho realizado no Serviço de Cirurgia Geral, Hospital das Clinicas da Universidade Federal de Pernambuco 


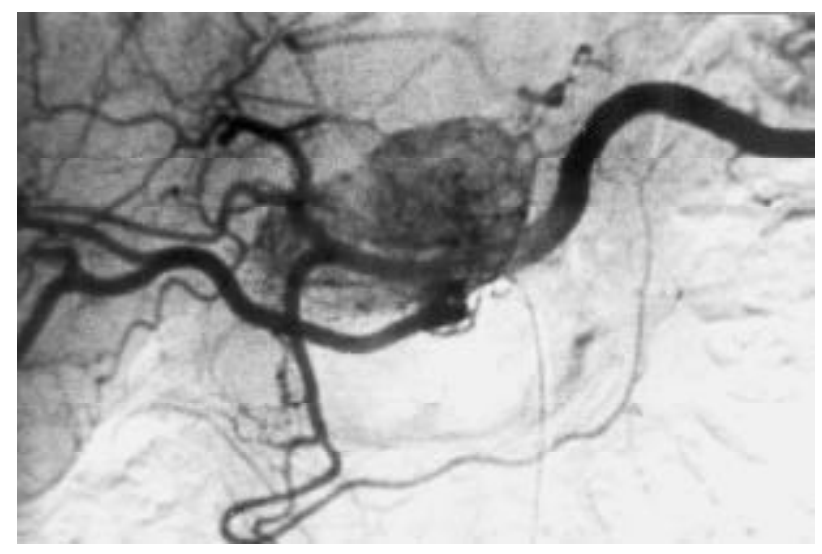

Figura 2 - Angiografia abdominal com injeção do tronco celíaco. Observe a hipervascularização da massa, achado esperado para o padrão hialino-vascular da doença de Castleman.

\section{DISCUSSÃO}

A DC é uma condição benigna, de etiologia desconhecida, caracterizada por proliferação de linfócitos maduros e células plasmáticas. Castleman em 1956 publicou os primeiros 13 casos dessa hiperplasia linfonodal gigante que se assemelhava a um timoma ${ }^{3}$.

Existem várias teorias para explicar os possíveis mecanismos patogênicos dessa lesão: inflama- ção crônica, processo hamartomatoso, estado de imunodeficiência e auto-imunidade.

Os achados histológicos podem ser divididos em dois tipos: 1. hialino-vascular e 2. celular plasmático. O tipo hialino-vascular é clinicamente assintomático, sendo encontrado em $90 \%$ dos casos. Já o celular plasmático, pode ser multicêntrico e caracteriza-se por sintomas como febre, anemia, velocidade de sedimentação elevada e hipergamaglobulinemia ${ }^{2}$.

Os achados radiológicos da DC abdominal são bastante inespecíficos. De acordo com Kim et $a l^{4}$, a forma localizada se caracteriza por uma grande massa, com ou sem linfadenomegalia associada, que apresenta pequena a moderada captação de contraste.

A DC é um tumor bastante incomum. A maioria das lesões intra-abdominais são localizadas na pelve, mesentério e regiao perinefrética ${ }^{5}$. Existe apenas um caso na literatura inglesa relatando a doença de Castleman simulando neoplasia pancreática. Tratava-se de uma mulher de 64 anos com uma lesão na cauda do pâncreas. De maneira semelhante ao caso aqui descrito, a paciente era oligosintomática e a ressecção foi curativa ${ }^{5}$.

Apesar de rara, a DC deve ser incluída no diagnostico diferencial das lesões pancreáticas atípicas.

\begin{abstract}
A 33-year-old man presented to our department with a 4-month history of right quadrant abdominal pain. Physical examination was normal. A chest $X$-ray showed no remarkable findings. Ultrasonography demonstrated a hypoechoic mass measuring $6 \mathrm{~cm}$ in the head of the pancreas. Computed tomography confirmed a solid mass in the pancreas without Wirsung or bile duct dilatation. At laparotomy, excision a $6 \mathrm{~cm}$ egg-shaped and hypervascular mass in the head of the pancreas was performed. Histologically, the features were consistent with Castleman disease. Castleman's disease is a rare, usually benign lymphoid condition described by Castleman (1954) and characterized by giant lymph nodes. Surgical resection is diagnostic and curative.
\end{abstract}

Key Words: Giant lymph node hyperplasia; Pancreas; Pancreatic neoplasms.

\section{REFERÊNCIAS}

1. Castleman B - Case records of The Massachusetts General Hospital. Case 4011. N Engl J Med, 1954, 250: 26-30.
2. Anagnostou D, Harrison CV - Angiofollicular lymph node hyperplasia (Castleman). J Clin Pathol 1972, 25(4):306-311.

3. Castleman B, Iverson L, Menendez VP - Localized mediastinal lymph-node hyperplasia resembling thymoma. Cancer, 1956, 9:822-830. 
4. Kim TJ, Han JK, Kim YH, et al. - Castleman disease of the abdomen: imaging spectrum and clinic pathologic correlations. J Comput Assist Tomogr, 2001, 25(2): 207-214

5. LeVan TA, Clifford S, Staren ED - Castleman's tumor masquerading as a pancreatic neoplasm. Surgery, 1989, 106(5): 884-887.
Endereço para correspondência: Antonio Cavalcanti de Albuquerque

Rua Deputado Pedro Pires Ferreira, 325/ 1601

Graças - Recife - PE

Brasil - CEP: 52050-480

Phone: (81) 99738223, (81) 32672670

E-mail: acam@elogica.com.br 